\title{
The Analysis of a SEIRS Epidemic Model with Time Delay on Complex Networks
}

\author{
Jing Dong, Tao Li*, Chen Wan, Xiongding Liu \\ School of Electronics and Information, Yangtze University, Jingzhou, China \\ Email: *taohust2008@163.com
}

How to cite this paper: Dong, J., Li, T., Wan, C. and Liu, X.D. (2017) The Analysis of a SEIRS Epidemic Model with Time Delay on Complex Networks. Open Access Library Journal, 4: e3901. https://doi.org/10.4236/oalib.1103901

Received: August 21, 2017

Accepted: September 11, 2017

Published: September 14, 2017

Copyright $\odot 2017$ by authors and Open Access Library Inc.

This work is licensed under the Creative Commons Attribution International License (CC BY 4.0).

http://creativecommons.org/licenses/by/4.0/

\section{(c) (i) Open Access}

\begin{abstract}
In this paper, a new epidemic SEIRS model with time delay on complex networks is proposed. Based on the mean field theory, the basic reproductive number and equilibriums of the model are derived. Moreover, the impact of the network topology and time delay on the basic reproductive number is analyzed. Theoretical analyses indicate that the basic reproductive number is dependent on the topology of the underlying networks. The time delay cannot change the basic reproductive number, but it can reduce the endemic level and weaken the epidemic spreading. The global asymptotically stability of the disease-free equilibrium and the permanence of epidemic are proved in detail. Numerical simulations confirm the analytical results.
\end{abstract}

\section{Subject Areas}

Network Modeling and Simulation

\section{Keywords}

Epidemic Model, Complex Networks, Time Delay, Permanence, Stability

\section{Introduction}

Mathematical models describing the population dynamics of infectious diseases have played an important role in better understanding epidemiological patterns and disease controls for a long time. The basis of modeling dynamics and evolution of infectious diseases are summarized by Anderson and May [1]. It is well known that the spread of a communicable disease involves disease-related factors such as infectious agent, mode of transmission, incubation periods, infectious periods, susceptibility, and resistance.

Continuous time deterministic epidemic models are traditionally elaborated as 
systems of ordinary differential equations. More realistic models should include some states of these systems, and ideally, a real system should be modeled by delay differential equation. Time delay plays an important part in propagation process of the epidemic, and we can simulate the exposed period of infectious diseases, the infections period of patients and the immunity period of recovery of the disease with time delay. However, compared with studies of the dynamical behaviors of the epidemic models with time delays on complex networks, only a few attentions about early studies have been paid to them on complex networks. In recent years, the dynamics of the $S I S, S I R, S E I$ and $S E I R$ epidemic models have received considerable attention [2] [3] [4] [5] [6]. In order to study the effects of disease latency or immunity in real life, the delay is incorporated in such models [7]-[12]. Liu and Deng et al. discussed epidemic SIS model with discrete time delay which represents the infectious period [13], and they obtained the basic reproduction number and discussed the persistence of the disease, but they failed to give the proof in detail. Wang and Wang et al. discussed an epidemic $S I R$ model with discrete time delay which represented the exposed period [14]. Based on the above, in this paper, we will present a suitable epidemic SEIRS model with time delay which represents the infectious period on complex networks using functional differential equations to investigate the epidemic spreading.

The rest of the paper is organized as follows: Section 2 derives a SEIRS model with time delay mechanism on scale-free networks. Then, Section 3 obtains two equilibriums and basic reproductive number. In Section 4, numerical simulations are performed. Finally, we conclude the paper in Section 5.

\section{Model Formulation}

One of the most effective interventions to contain the spread of epidemic diseases is the delay mechanism as discussed above. In order to investigate the efficiency of delay mechanism, we consider the new SEIRS model with delay mechanism on complex networks. On the complex networks, each individual is represented by a node of the network and the edges are the connections between individuals along which the infection may spread. Taking the connectivity among different nodes into consideration, let $S_{k}(t), E_{k}(t), I_{k}(t)$ and $R_{k}(t)$ be the relative densities of susceptible, exposed, infected and recovered nodes of degree $k$ at time $t$ respectively. In the course of disease transmission, a susceptible individual will be infected with probability $\beta_{1}\left(\beta_{2}\right)$ if it connects to a exposed (infected) one. $r$ is the state transition rate from exposed to infectious individual. The rate constant of recovery for infected individuals is denoted by $\alpha$. $\tau$, for recovered nodes, is the effectively constant of immunity, and there are still recovered nodes, when $t$ moment from $t-\tau$ moment, which will lose the immunity ability to become the susceptible nodes again. Here, we assume that the birth rate equals the death rate, and the rate constant is $l$. Thus, the dynamic mean-field reaction rate equations can be written as 


$$
\left\{\begin{array}{l}
\frac{\mathrm{d} S_{k}(t)}{\mathrm{d} t}=l-l S_{k}(t)-k \beta_{1} \Theta_{1}(t) S_{k}(t)-k \beta_{2} \Theta_{2}(t) S_{k}(t)+\alpha I_{k}(t-\tau) \mathrm{e}^{-l \tau} \\
\frac{\mathrm{d} E_{k}(t)}{\mathrm{d} t}=k \beta_{1} \Theta_{1}(t) S_{k}(t)+k \beta_{2} \Theta_{2}(t) S_{k}(t)-(l+r) E_{k}(t) \\
\frac{\mathrm{d} I_{k}(t)}{\mathrm{d} t}=r E_{k}(t)-(l+\alpha) I_{k}(t) \\
\frac{\mathrm{d} R_{k}(t)}{\mathrm{d} t}=\alpha I_{k}(t)-\alpha I_{k}(t-\tau) \mathrm{e}^{-l \tau}-l R_{k}(t)
\end{array}\right.
$$

where the probability $\Theta_{1}(t)$ denotes a link pointing to an exposed individual, and satisfies

$$
\Theta_{1}(t)=\frac{\sum_{k} k P(k) E_{k}(t)}{\sum_{s} s P(s)}=\frac{1}{\langle k\rangle} \sum_{k} k P(k) E_{k}(t) .
$$

The probability $\Theta_{2}(t)$ denotes a link pointing to an infected individual, and satisfies

$$
\Theta_{2}(t)=\frac{\sum_{k} k P(k) I_{k}(t)}{\sum_{s} s P(s)}=\frac{1}{\langle k\rangle} \sum_{k} k P(k) I_{k}(t) .
$$

Where $\langle k\rangle$ describes the average degree and $P(k)$ describes the connectivity distribution. $E(t)=\sum_{k} P(k) E_{k}(t)$ is the total density of exposed individuals, and the $I(t)=\sum_{k} P(k) I_{k}(t)$ is the total density of infected individuals in the whole network.

For simplicity, through this paper, let $\rho(t)=\beta_{1} \Theta_{1}(t)+\beta_{2} \Theta_{2}(t)$ and $P_{k}=k P(k) /\langle k\rangle$. Thus, the system (1) is equivalent to the following model

$$
\left\{\begin{array}{l}
\frac{\mathrm{d} S_{k}(t)}{\mathrm{d} t}=l-l S_{k}(t)-k \rho(t) S_{k}(t)+\alpha I_{k}(t-\tau) \mathrm{e}^{-l \tau} \\
\frac{\mathrm{d} E_{k}(t)}{\mathrm{d} t}=k \rho(t) S_{k}(t)-(l+r) E_{k}(t) \\
\frac{\mathrm{d} I_{k}(t)}{\mathrm{d} t}=r E_{k}(t)-(l+\alpha) I_{k}(t) \\
\frac{\mathrm{d} R_{k}(t)}{\mathrm{d} t}=\alpha I_{k}(t)-\alpha I_{k}(t-\tau) \mathrm{e}^{-l \tau}-l R_{k}(t)
\end{array}\right.
$$

With the normalization condition, there is

$$
S_{k}(t)+E_{k}(t)+I_{k}(t)+R_{k}(t)=1 .
$$

Obviously, the initial conditions for system satisfy

$$
0 \leq S_{k}(0), E_{k}(0), I_{k}(0), R_{k}(0) \leq 1 .
$$

\section{Analysis of the Novel SEIRS Model}

In this part, we put forward the analytic solution of system (2) for describing the dynamic behavior.

Theorem 1. Denote

$$
R_{0}=\frac{\left[\beta_{1}(l+\alpha)+r \beta_{2}\right]\left\langle k^{2}\right\rangle}{(l+\alpha)(l+r)\langle k\rangle} .
$$


The disease-free equilibrium $E_{0}(1,0,0,0)$ of system (2) always exists and there exists a positive endemic equilibrium $E_{+}\left(S_{k}^{\infty}, E_{k}^{\infty}, I_{k}^{\infty}, R_{k}^{\infty}\right)$ of system (2) when $R_{0}>1$.

Proof. To get the equilibrium solution $E_{+}\left(S_{k}^{\infty}, E_{k}^{\infty}, I_{k}^{\infty}, R_{k}^{\infty}\right)$, we need to make the right side of system (2) equal to zero. Then, the equilibrium $E_{+}\left(S_{k}^{\infty}, E_{k}^{\infty}, I_{k}^{\infty}, R_{k}^{\infty}\right)$ should satisfy

$$
\left\{\begin{array}{l}
l+\alpha I_{k}^{\infty} \mathrm{e}^{-l \tau}-k \rho^{\infty} S_{k}^{\infty}-l S_{k}^{\infty}=0 \\
k \rho^{\infty} S_{k}^{\infty}-(l+r) E_{k}^{\infty}=0 \\
r E_{k}^{\infty}-(l+\alpha) I_{k}^{\infty}=0 \\
\alpha I_{k}^{\infty}-\alpha I_{k}^{\infty} \mathrm{e}^{-l \tau}-l R_{k}^{\infty}=0 .
\end{array}\right.
$$

Thus, we have

$$
\left\{\begin{array}{l}
S_{k}^{\infty}=\frac{l+\alpha I_{k}^{\infty} \mathrm{e}^{-l \tau}}{l+k \rho} \\
E_{k}^{\infty}=\frac{l+\alpha}{r} I_{k}^{\infty} \\
R_{k}^{\infty}=\frac{\alpha-\alpha \mathrm{e}^{-l \tau}}{l} I_{k}^{\infty}
\end{array}\right.
$$

According to the normalization condition $S_{k}+E_{k}+I_{k}+R_{k}=1$, we can get

$$
\left\{\begin{array}{l}
S_{k}^{\infty}=\frac{\left(r l^{2}+k \rho r l\right) \alpha \mathrm{e}^{-l \tau}+l(l+k \rho)\left[l(l+\alpha)+\alpha r\left(1-\mathrm{e}^{-l \tau}\right)+r l\right]}{B_{k}} \\
E_{k}^{\infty}=\frac{(l+\alpha) k \rho l}{B_{k}} \\
I_{k}^{\infty}=\frac{k \rho r l}{B_{k}} \\
R_{k}^{\infty}=\frac{\left(1-\mathrm{e}^{-l \tau}\right) \alpha k \rho r}{B_{k}}
\end{array}\right.
$$

where $B_{k}=r l \alpha \mathrm{e}^{-l \tau}+(l+k \rho)\left[l(l+\alpha)+\alpha r\left(1-\mathrm{e}^{-l \tau}\right)+r l\right]$.

For $\rho=\beta_{1} \Theta_{1}(t)+\beta_{2} \Theta_{2}(t)=\langle k\rangle^{-1} \sum_{k} k P(k)\left(E_{k} \beta_{1}+I_{k} \beta_{2}\right) \leq \beta_{1}+\beta_{2}$, we can find that $\rho=0$ satisfies Equation (5). Therefore, $S_{k}=1$ and $E_{k}=I_{k}=R_{k}=0$ is a disease-free equilibrium of system (2). Substituting $I_{k}$ and $E_{k}$ of Equation (5) into $\rho$, we can obtain

$$
\rho=\frac{\sum_{k} k^{2} P(k)}{\langle k\rangle} \frac{l \rho\left[r \beta_{2}+(l+\alpha) \beta_{1}\right]}{B_{k}} .
$$

We suppose

$$
f(\rho)=1-\frac{\sum_{k} k^{2} P(k)}{B_{k}} \frac{l\left[r \beta_{2}+(l+\alpha) \beta_{1}\right]}{\langle k\rangle},
$$

so that, Equation (6) can be written

$$
\rho f(\rho)=0 .
$$


Due to $\frac{\mathrm{d} f(\rho)}{\mathrm{d} \rho}>0$ and $f\left(\beta_{1}+\beta_{2}\right)>0$, the equation $f(\rho)=0$ has a non-trivial solution if and only if $f(0)<0$.

So, we have

$$
\frac{\beta_{1}(l+\alpha)+r \beta_{2}}{(l+\alpha)(l+r)} \frac{\sum_{k} k^{2} P(k)}{\langle k\rangle}>1 .
$$

Let's define $\left\langle k^{2}\right\rangle=\sum_{k} k^{2} P(k)$, thus, we can compute the base reproduction number as follows

$$
R_{0}=\frac{\left[\beta_{1}(l+\alpha)+r \beta_{2}\right]\left\langle k^{2}\right\rangle}{(l+\alpha)(l+r)\langle k\rangle} .
$$

Namely, there is the unique nontrivial solution if and only if $R_{0}>1$. From Equations ((4) and (5)), we are able to obtain

$$
0<S_{k}^{\infty}<1,0<E_{k}^{\infty}<1, \quad 0<I_{k}^{\infty}<1, \quad 0<R_{k}^{\infty}<1 .
$$

So, we define the equilibrium $E_{+}\left(S_{k}^{\infty}, E_{k}^{\infty}, I_{k}^{\infty}, R_{k}^{\infty}\right)$. Hence, when $R_{0}>1$, system (2) has a unique positive endemic equilibrium $E_{+}\left(S_{k}^{\infty}, E_{k}^{\infty}, I_{k}^{\infty}, R_{k}^{\infty}\right)$. The proof is completed.

Remark. The basic reproductive number is obtained by (7), which depends on different model parameters and fluctuations of the degree distribution. The delay parameter cannot change the basic reproductive number. Clearly, in the eco-limits of infinite size network the number of nodes grows to infinity, i.e., $N \rightarrow \infty$, then $\frac{\left\langle k^{2}\right\rangle}{\langle k\rangle}$ grows to infinity, so the increase of the basic reproductive number, i.e., $R_{0} \rightarrow \infty$, is obvious.

Theorem 2. If $R_{0}<1$, the disease-free equilibrium $E_{0}$ is globally asymptotically stable. When $R_{0}>1$, the epidemic disease is permanent, which means there exists $\xi>0$, such that

$$
\liminf _{t \rightarrow \infty}\left\{S_{k}(t), E_{k}(t), I_{k}(t), R_{k}(t)\right\}_{k=1}^{n} \geq \xi
$$

where $\left(S_{k}(t), E_{k}(t), I_{k}(t)\right)$ is any solution of system (2), satisfying $E_{k}(0)>0$ or $I_{k}(0)>0$.

Proof. For $S_{k}(t)+E_{k}(t)+I_{k}(t)+R_{k}(t)=1$, i.e., the values of $S_{k}(t), E_{k}(t)$ and $I_{k}(t)$ are fixed, a unique corresponding $R_{k}(t)$ exists. We can discuss the first three equations of system (2) with $R_{k}(t)=1-S_{k}(t)-E_{k}(t)-I_{k}(t)$ at all $t$. System (2) satisfies

$$
\left\{\begin{array}{l}
\frac{\mathrm{d} E_{k}(t)}{\mathrm{d} t}=k \rho\left(1-E_{k}(t)-I_{k}(t)-R_{k}(t)\right)-(l+r) E_{k}(t) \\
\frac{\mathrm{d} I_{k}(t)}{\mathrm{d} t}=r E_{k}(t)-(l+\alpha) I_{k}(t) \\
\frac{\mathrm{d} R_{k}(t)}{\mathrm{d} t}=\alpha I_{k}(t)-l R_{k}(t)-\alpha I_{k}(t-\tau) \mathrm{e}^{-l \tau}
\end{array}\right.
$$


Thus, the jacobian matrix of the virus-free equilibrium for the system (2) can be obtained, the jacobian matrix is a $3 n \times 3 n$ matrix, such that

$$
\begin{aligned}
& J=\left(\begin{array}{ccc}
A_{11} & \ldots & A_{1 n} \\
\vdots & \ddots & \vdots \\
A_{n 1} & \cdots & A_{n n}
\end{array}\right), \\
& A_{11}=\left(\begin{array}{ccc}
-(l+r)+k \beta_{1} P_{1} & k \beta_{2} P_{1} & 0 \\
r & -(l+\alpha) & 0 \\
0 & \alpha\left(1-\mathrm{e}^{-l \tau}\right) & -l
\end{array}\right) \text {, } \\
& A_{1 n}=\left(\begin{array}{ccc}
k \beta_{1} P_{n} & k \beta_{2} P_{n} & 0 \\
0 & 0 & 0 \\
0 & 0 & 0
\end{array}\right), \\
& A_{n 1}=\left(\begin{array}{ccc}
n k \beta_{1} P_{1} & n k \beta_{2} P_{1} & 0 \\
0 & 0 & 0 \\
0 & 0 & 0
\end{array}\right), \\
& A_{n n}=\left(\begin{array}{ccc}
-(l+r)+k \beta_{1} P_{n} & n \beta_{2} P_{n} & 0 \\
r & -(l+\alpha) & 0 \\
0 & \alpha\left(1-\mathrm{e}^{-l \tau}\right) & -l
\end{array}\right) .
\end{aligned}
$$

We can put forward the characteristic equation of the disease-free equilibrium from jacobian matrix, such that

$$
(\lambda+l+\alpha)^{n-1}(\lambda+l+r)^{n-1}\left(\lambda^{2}+p \lambda+q\right)=0,
$$

where $p=(l+r)+(l+\alpha)-n \beta_{1} P_{n}$ and $q=(l+\alpha)(l+r)-\left[(l+\alpha) \beta_{1}+r \beta_{2}\right] n P_{n}$.

Note that $R_{0}<1$ is equivalent to $q>0$ which $(l+r)(l+\alpha)-\left[(l+\alpha) \beta_{1}+r \beta_{2}\right] \sum_{i=1}^{n} i P_{i}>0$, so that $(l+r)(l+\alpha)>\left[(l+\alpha) \beta_{1}+r \beta_{2}\right] \sum_{i=1}^{n} i P_{i}$, which means that $p>0$. Therefore, the eigenvalue $\lambda$ of $J$ are all negative if $R_{0}<1$; otherwise, if and only if $R_{0}<1$, here exists a unique positive eigenvalue $\lambda$ of $J$. Using the Perron-Feobenius theorem, this suggests that the maximal real part of all eigenvalues of $J$ is positive only if $R_{0}>1$. Thus, we can obtain the results of this theorem by using a theorem presented of Lajmanovich and York [15]. The proof is completed.

\section{Numerical Simulations and Sensitivity Analysis}

To support and explain our theoretical analysis results, we present several numerical simulations in this section. The system (2) is considered on complex networks, whose the degree distribution is $P(k)=c k^{-3}$, where the parameter satisfies $\sum_{k=1}^{n} c k^{-3}=1, n=1000$. We suppose $S_{k}(0)=0.9, E_{k}(0)=0.1$, 
$I_{k}(0)=0$ and $R_{k}(0)=0$.

In Figure 1 , the parameters are chosen as $\beta_{1}=0.01, \beta_{2}=0.1, l=0.2$, $u=0.4, r=0.5$, thus the threshold value $R_{0}=0.4230<1$. According to $R_{0}<1$, we can see that when $R_{0}<1,\left(E_{k}+I_{k}\right)$ grows to zero, i.e., the infectious individuals will ultimately disappear. If $R_{0}<1$, the equilibrium $E_{0}$ is globally attractive and the disease eventually disappear.

In Figure 2, the parameters are chosen as $\beta_{1}=0.3, \beta_{2}=0.4, l=0.2$, $u=0.4, r=0.5$, thus the threshold value $R_{0}=2.8705>1$. We can see that when $R_{0}>1,\left(E_{k}+I_{k}\right)$ grows to a constant, i.e., the epidemic disease is permanent and the number of infected individuals will converge to a positive constant.

In Figure 3, the parameters are chosen as $\beta_{1}=0.01, \beta_{2}=0.1, l=0.2$, $u=0.4, r=0.5, R_{0}=0.4230$. We can see that the corresponding $\left(E_{k}+I_{k}\right)$

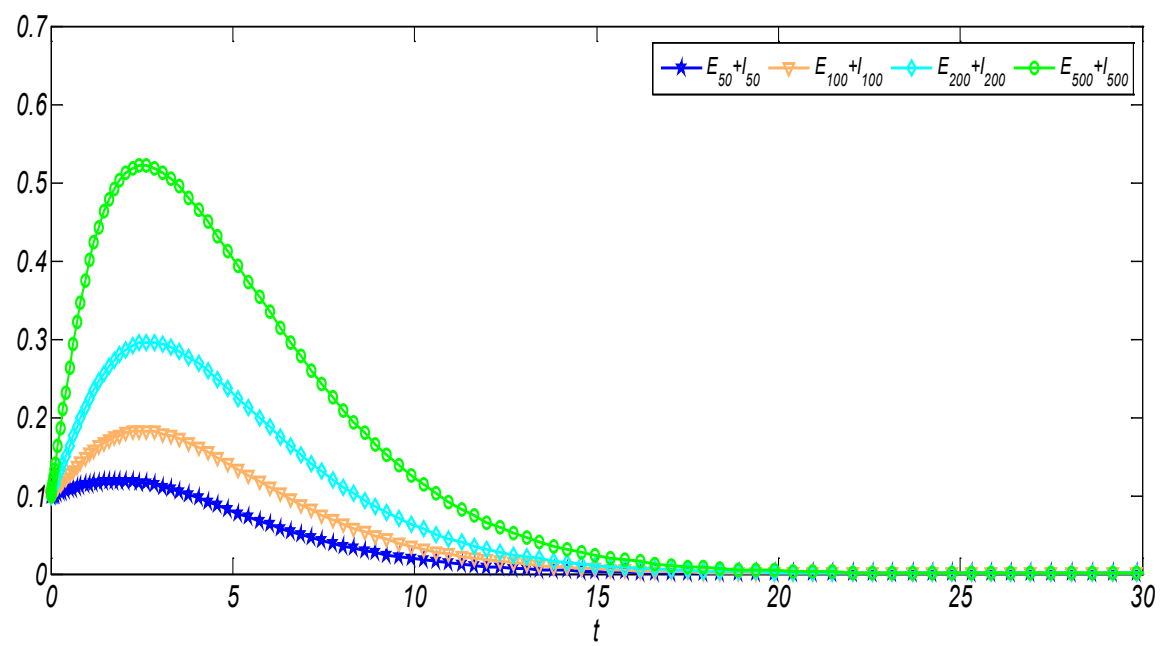

Figure 1. With $R_{0}=0.4230<1$, the prevalence of $E_{k}(t)$ and $I_{k}(t)$ versus $t$ corresponding for $k=50,100,200,500$.

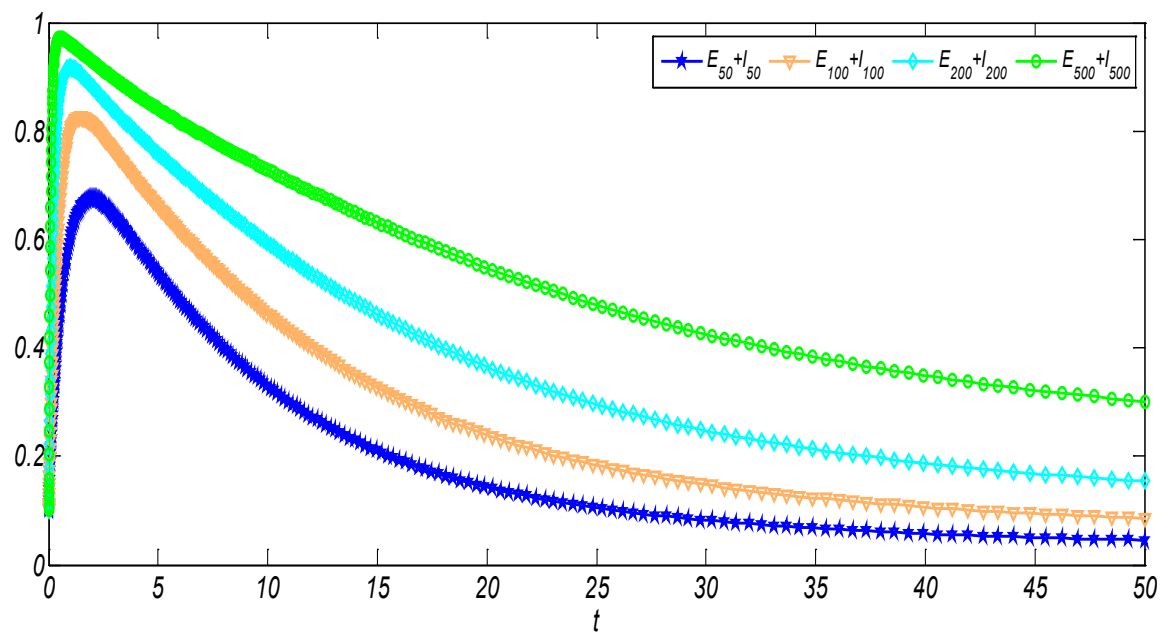

Figure 2. With $R_{0}=2.8705>1$, the prevalence of $E_{k}(t)$ and $I_{k}(t)$ versus $t$ corresponding for $k=50,100,200,500$. 


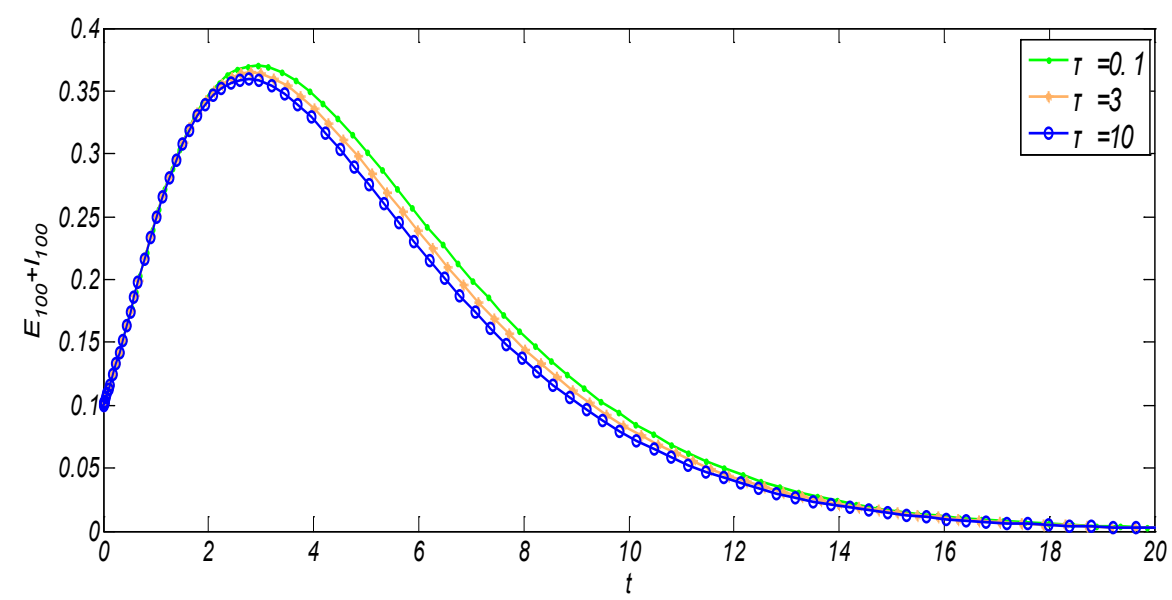

Figure 3. With $R_{0}<1$, the prevalence of $E_{k}(t)$ and $I_{k}(t)$ versus $t$ corresponding for $k=100$ and different parameter $\tau$.

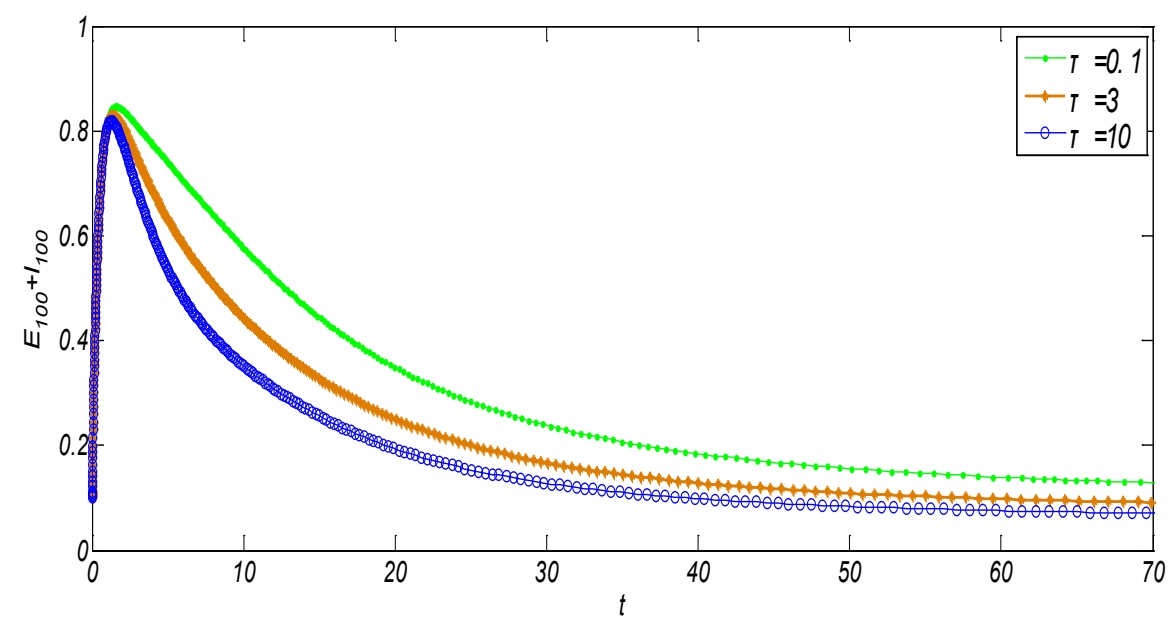

Figure 4. With $R_{0}>1$, the prevalence of $E_{k}(t)$ and $I_{k}(t)$ versus $t$ corresponding for $k=100$ and different parameter $\tau$.

decreases significantly as the delay parameter $\tau$ increases, i.e., a larger delay parameter can weaken the spreading of disease. The larger delay is, the lower corresponding $\left(E_{k}+I_{k}\right)$ level is.

In Figure 4 , the parameters are chosen as $\beta_{1}=0.3, \beta_{2}=0.4, l=0.2$, $u=0.4, r=0.5, R_{0}=0.4230$. We can see that the corresponding $\left(E_{k}+I_{k}\right)$ decreases significantly as the delay parameter $\tau$ increases, i.e., a larger delay parameter can reduce the endemic level.

\section{Conclusion}

In this paper, a novel SEIRS epidemic model of delay mechanism on complex networks has been presented. Using the field theory, we calculated two epidemiologically relevant quantities, i.e., the basic reproductive number for invasion and the endemic prevalence of epidemic diseases. We have proved that if the basic reproductive number $R_{0}<1$, the disease-free equilibrium is globally asymp- 
totically stable; while $R_{0}>1$, the endemic equilibrium is permanent. Moreover, increasing delay parameters can result in the weakness of the diseases spreading and the decrease of population infected. Numerical simulations show that the endemic equilibrium $E_{+}$is globally asymptotically stable when $R_{0}>1$ (as shown in Figure 2). It is interesting but challenging to discuss the stability of equilibrium $E_{+}$. We leave it for our future work. This study has valuable guiding significance in effectively predicting epidemic spreading.

\section{Acknowledgements}

This work is supported in part by the National Natural Science Foundation of China under Grants 61672112, the Teaching Research Project of Hubei Province under Grant 2014260, and the Teaching Research Project of Yangtze University under Grant JY2015010.

\section{References}

[1] Anderson, R.M. and May, R.M. (1991) Infectious Diseases of Humans: Dynamics and Control. Oxford University Press Inc., New York.

[2] Wei, J. and Zou, X. (2006) Bifurcation Analysis of a Population Model and the Resulting SIS Epidemic Model with Delay. Journal of Computational and Applied Mathematics, 197, 169-187. https://doi.org/10.1016/j.cam.2005.10.037

[3] Huang, G., Takeuchi, Y., Ma, W., et al. (2010) Global Stability for Delay SIR and SEIR Epidemic Models with Nonlinear Incidence Rate. Bulletin of Mathematical Biology, 72, 1192-1207. https://doi.org/10.1007/s11538-009-9487-6

[4] Xiao, Y., Chen, L. and ven den Bosch, F. (2002) Dynamical Behavior for a Stage-Structured SIR Infectious Disease Model. Nonlinear Analysis. Real World Applications, 3, 175-190. https://doi.org/10.1016/S1468-1218(01)00021-9

[5] Gao, S., Chen, L. and Teng, Z. (2007) Impulsive Vaccination of an SEIRS Model with Time Delay and Varying Total Population Size. Bulletin of Mathematical Biology, 69, 731-745. https://doi.org/10.1007/s11538-006-9149-x

[6] Li, T., Liu, X.D., Wu, J., et al. (2016) An Epidemic Spreading Model on Adaptive Scale-Free Networks with Feedback Mechanism. Physica A: Statistical Mechanics and Its Applications, 450, 649-656. https://doi.org/10.1016/j.physa.2016.01.045

[7] Li, J. and Ma, Z. (2006) Analysis of Two SEIS Epidemic Models with Fixed Period of Latency. Journal of Systems Science and Mathematical Sciences, 26, 228-236.

[8] Enatsu, Y., Nakata, Y. and Muroya, Y. (2012) Global Stability of SIRS Epidemic Models with a Class of Nonlinear Incidence Rates and Distributed Delays. Acta Mathematica Scientia, 32, 851-865. https://doi.org/10.1016/S0252-9602(12)60066-6

[9] Nakata, Y. (2011) On the Global Stability of a Delayed Epidemic Model with Transport-Related Infection. Nonlinear Analysis. Real World Applications, 12, 3028-3034. https://doi.org/10.1016/j.nonrwa.2011.05.004

[10] Zhang, T. and Teng, Z. (2008) Global Behavior and Permanence of SIRS Epidemic mOdel with Time Delay. Nonlinear Analysis: Real World Applications, 9, 1409-1424. https://doi.org/10.1016/j.nonrwa.2007.03.010

[11] Liu, Q., Jiang, D., Shi, N., et al. (2016) Asymptotic Behavior of a Stochastic Delayed SEIR Epidemic Model with Nonlinear Incidence. Physica A: Statistical Mechanics and Its Applications, 462, 870-882. 
[12] Sharma, N. and Gupta, A.K. (2017) Impact of Time Delay on the Dynamics of SEIR Epidemic Model using Cellular Automata. Physica A: Statistical Mechanics and Its Applications, 471, 114-125.

[13] Liu, Q.M., Deng, C.S. and Sun, M.C. (2014) The Analysis of an Epidemic Model with Time Delay on Scale-Free Networks. Physica A: Statistical Mechanics and Its Applications, 410, 79-87.

[14] Wang, J., Wang, J., Liu, M., et al. (2014) Global Stability Analysis of an SIR Epidemic Model with Demographics and Time Delay on Networks. Physica A: Statistical Mechanics and Its Applications, 410, 268-275.

[15] Lajmanovich, A. and Yorke, J.A. (1976) A Deterministic Model for Gonorrhea in a Nonhomogeneous Population. Mathematical Biosciences, 28, 221-236.

Submit or recommend next manuscript to OALib Journal and we will provide best service for you:

- Publication frequency: Monthly

- 9 subject areas of science, technology and medicine

- Fair and rigorous peer-review system

- Fast publication process

- Article promotion in various social networking sites (LinkedIn, Facebook, Twitter, etc.)

- Maximum dissemination of your research work

Submit Your Paper Online: Click Here to Submit

Or Contact service@oalib.com 